\title{
La voix endeuillée : une lecture des « Grands moments d'un chanteur » de Louis-René des Forêts
}

\section{Midori Ogawa}

\section{OpenEdition}

12 Journals

\section{Édition électronique}

URL : http://journals.openedition.org/recherchestravaux/449

DOI : 10.4000/recherchestravaux.449

ISSN : 1969-6434

\section{Éditeur}

UGA Éditions/Université Grenoble Alpes

\section{Édition imprimée}

Date de publication : 15 mai 2011

Pagination : 81-96

ISBN : 978-2-84310-200-4

ISSN : 0151-1874

\section{Référence électronique}

Midori Ogawa, "La voix endeuillée : une lecture des « Grands moments d'un chanteur » de Louis-René des Forêts », Recherches \& Travaux [En ligne], 78 | 2011, mis en ligne le 15 novembre 2012, consulté le 07 septembre 2020. URL : http://journals.openedition.org/recherchestravaux/449 ; DOI : https:// doi.org/10.4000/recherchestravaux.449 
Midori Ogawa

Université de Tsukuba (Japon)

\section{La voix endeuillée : une lecture des "Grands moments d'un chanteur» de Louis-René des Forêts}

«L'amour d'une patrie qui n'existe plus est une passion sans remède."

Balzac

Louis-René des Forêts est un écrivain discret et peu prolifique. Il a débuté sa carrière pendant la Résistance en publiant d'abord des chroniques littéraires et musicales. "Les grands moments d'un chanteur» ont été d'abord publiés entre 1953 et 1954 et repris dans un recueil de nouvelles intitulé La Chambre des Enfants en $1960^{\mathrm{I}}$. Outre le ton désenchanté, on est étonné par la manière étrange dont la musique y est présentée : en effet, elle donne son éclat particulier dans sa propre négation. Même si la musique n'est pas ici l'objet d'une critique, elle est totalement sujette à caution.

\section{L'idée de la musique à l'aube du postromantisme}

Si tous les discours musicaux s'inscrivent dans l'évolution du concept que nous avons de la musique, le récit de des Forêts doit lui-même refléter cette histoire riche des idées musicales. C'est à l'époque romantique, on s'en souvient,

I. L.-R. des Forêts, La Chambre des Enfants, Gallimard, coll. «L'Imaginaire», I960. Ce recueil inclut quatre nouvelles, "Les grands moments d'un chanteur» (désormais GMC), «La chambre des enfants», "Une mémoire démentielle», "Dans un miroir», qui sont en rapport étroit entre eux. Il serait donc souhaitable, après une lecture individuelle de chacune des nouvelles, de remettre ces pièces dans l'ensemble de l'ouvrage. «Les grands moments d'un chanteur» ont été publiés pour la première fois dans la revue Les Lettres Nouvelles, ${ }^{\circ}$ Io (déc. I953) et $n^{\circ}$ II (janv. 1954). 
que la musique a été tenue en la plus haute estime, comme le montre ce passage significatif de «Massimilla Doni» qui en dit long:

La musique moderne, qui veut une paix profonde, est la langue des âmes tendres, amoureuses, enclines à une noble exaltation intérieure. Cette langue, mille fois plus riche que celle des mots, est au langage ce que la pensée est à la parole [...] Les autres arts imposent à l'esprit des créations définies, la musique est infinie dans les siennes².

À cette acception romantique qui surévalue le statut de la musique s'opposera le discours postromantique - dont celui de des Forêts - qui trouve une des raisons d'être dans sa capacité de la remettre en question.

En 1924, Franz Kafka rédigea un récit intitulé «Joséphine la cantatrice ou le Peuple des souris» que l'on peut considérer comme une revanche sur la pensée romantique ${ }^{3}$. L'héroïne, Joséphine, est une souris qui prétend chanter l'âme du peuple de sa tribu alors qu'elle ne fait que siffler en réalité. À travers ce récit allégorique, Kafka affirme la disparition du chant comme poésie (le chant est ici associé à une nostalgie), et en même temps, il dénonce le dispositif théâtral qui dissimule cette disparition. La diva Joséphine recourt à tous les artifices théâtraux afin qu'il y ait, sur la scène vidée de toute inspiration, un événement qu'elle prétend être le chant. Cette tentative de faire croire à l'existence du chant là où il n'y en a pas dévoile le mensonge lié à l'acte de création et dénonce l'idée de la musique suprême, unique et absolue.

Sans aller jusqu'à oser douter de l'existence de la musique, Balzac, déjà dans son "Sarrasine», a fait un pas décisif en mettant en exergue le paraitre qui se mêle au phénomène musical ${ }^{4}$. Zambinella est un castrat dont Sarrasine, héros et sculpteur talentueux, est follement épris. Sarrasine étant la proie d'un leurre inventé et entretenu par l'acte de la création (Zambinella, un homme, produit une musique féminine ou du moins efféminée), son histoire révèle un paradoxe, car le héros croit le monde plus vrai lorsqu'il vit dans une pure illusion :

Cette voix empreinte de faiblesse, l'attitude, les manières et les gestes de Zambinella, marqués de tristesse, de mélancolie, et de découragement, réveillaient dans son âme [de Sarrasine] toutes les richesses de la passion. (p. IO70)

À la fin du récit, Sarrasine déclare : «Sans cesse je penserai à cette femme imaginaire en voyant une femme réelle»(p. I074). Mais ce qui est plus frappant ici, c'est que Zambinella aussi est victime d'un leurre dont il est

2. H. de Balzac, «Massimilla Doni», dans La Comédie humaine, t. X (Études philosophiques), Gallimard, coll. «La Pléiade», 1979, p. 587-578.

3. Fr. Kafka, «Joséphine la cantatrice ou le Peuple des souris», dans Euvres complètes, t. II, Gallimard, coll. "La Pléiade», 1988, p. 774-790.

4. H. de Balzac, "Sarrasine», dans La Comédie humaine, t. VI (Études de mours, Scènes de la vie parisienne), Gallimard, coll. «La Pléiade», I977, p. I043-I076. 
involontairement l'agent. La voix de Zambinella, puissante et enchanteresse puisque artificiellement transformée, fait illusion sur le reste de son corps, à l'exception de son âme qui reste telle qu'elle est. Cette division de l'être entre le corps et l'esprit, révélée dans le personnage de Zambinella, nous amène à nous interroger sur la notion d'identité et d'identification à travers le thème de la voix transformée.

Le discours postromantique ne se limite pas à critiquer, d'une manière ou d'une autre, l'idée idéalisée de musique considérée jusque-là comme substance pure, ou dans une acception mégalomaniaque, comme art des arts. Comme le montrent les exemples ci-dessus, le discours sur la musique a déplacé son accent, celui-ci étant désormais porté moins sur l'objet lui-même que sur son rapport à l'auditeur. C'est dans ce contexte que la voix attire l'attention, d'autant plus qu'elle se situe entre notre corps et la musique. La musique a ainsi cessé d'être autonome et indépendante : elle nous intéresse dans la mesure où elle questionne notre être et notre rapport au monde. À travers cet aspect relationnel sont également mis en avant l'auditeur (dont la naïveté est souvent l'objet d'accusations chez Balzac)'s, ainsi que le dispositif par lequel la musique nous est présentée. En ce sens, le discours postromantique sur la musique se montre plus complexe et ambigu. Cela est d'autant plus vrai lorsqu'il s'agit du récit de Louis-René des Forêts qui nous déconcerte à plus d'un titre. Et cette impression tient non seulement à la narration obscure qui s'appuie sur "un principe d'incertitude ${ }^{6} »$ qui laisse libre cours à des interprétations multiples, mais aussi à deux procédés contradictoires qu'adopte ce récit. En effet, «Les grands moments d'un chanteur» empruntent au conte son modèle - en particulier, la parenté avec les contes romantiques est évidente, on y reviendra - avec l'intention de présenter l'histoire comme une fiction allégorique, en même temps qu'ils optent pour la narration à la première personne qui annule la distance entre le sujet de l'énonciation et son objet, ce qui engendre un effet de malaise profond, voire de perversion. C'est comme si un sujet moderne était pris dans un monde romantique, la fusion avec la musique devenant la source d'un cauchemar qui n'en finit plus. L'ironie de des Forêts consiste à montrer davantage l'aspect néfaste de l'enchantement musical qui nuit et détruit les personnages.

5. Dans «Gambara» par exemple, le jeune comte italien déplore la folie de la jeune fille qui sacrifie tout pour son mari compositeur qui se croit un génie. L'orgueil démesuré et le génie incompris du compositeur n'échappent ici évidemment pas à une critique, mais l'auteur accuse en même temps la naïveté de sa femme rendue folle par la musique : "Marianna ne put retenir ses pleurs. [...] Un horrible sourire effleura les lèvres de Marianna. Le comte fut épouvanté par la naïveté de cette folie» (H. de Balzac, "Gambara», dans La Comédie bumaine, t. X, op. cit., p. 492.)

6. J. Roudaut, Louis-René des Forêts, Seuil, coll. «Les Contemporains», I995, p. I24. 


\section{Un conte romantique après l'heure?}

Cette attitude attribue au récit un caractère allégorique, moral et philosophique, à l'exemple des contes balzaciens où des personnages font face à des questions essentielles mises en relation avec l'œuvre d'art. Cette facture, riche en exemples à l'époque romantique, donne un modèle au récit de des Forêts. Je propose ici de le comparer avec trois contes romantiques, afin de mieux cerner sur quel fond repose ce récit d'apparence hermétique. Mais avant, voici un résumé du récit :

Un narrateur relate l'incroyable succès d'un musicien, modeste, qui devient de façon imprévisible un exceptionnel chanteur et qui, de façon tout aussi inconcevable à ceux qui l'admirent, renonce à tout. Malgré ses efforts pour être précis, le narrateur ne peut développer son récit que d'hypothèse en hypothèse : les motivations sont incertaines, les causes cachées. (Roudaut, p. I23.)

Tout est, en effet, totalement sujet à caution dans l'histoire qui nous est contée : de la première apparition sur scène, où le joueur de hautbois se métamorphose subitement en incarnation parfaite du libertin diabolique, jusqu'au sabotage final qui a lieu, par un effet de boucle trop parfaite, lors d'une autre représentation du Don Giovanni de Mozart. La fulgurante carrière du chanteur reste donc doublement marquée par le mystère : doit-elle quoi que ce soit à la volonté de Molieri qui aurait pu manigancer l'agression contre celui qu'il remplace avec une extraordinaire facilité, et qui paraît, aux yeux du narrateur, avoir choisi de se suicider de son propre chef pour retrouver l'anonymat de la fosse d'orchestre?

Durant toutes les actions qui s'enchaînent depuis le succès inattendu et inespéré jusqu'au moment où le héros démolit sa réputation et sa vie d'artiste par un sabotage anarchique, un doute reste suspendu : à quoi tient cette carrière à la fois si extraordinaire et si étrange? Cette question s'enchaine à une autre : qui est Frédéric Molieri, un heureux élu du Ciel, un vainqueur vaincu, un homme infortuné malmené par le destin ou encore un charlatan? Ce serait trop improbable, si l'on réduisait le destin de Frédéric Molieri à sa seule volonté. Que s'est-il passé en vérité dans ses aventures? C'est dans ces questionnements que le texte de des Forêts montre sa parenté la plus évidente avec le conte fantastique. Sorte de mythe faustien, «Les grands moments d'un chanteur» évoquent un autre conte fantastique allemand dans lequel le héros obtient un pouvoir démoniaque en échange de sa propre ombre. Il s'agit de L'Étrange Histoire de Peter Schlemihl de Chamisso ${ }^{8}$. Le rapprochement est plus que fortuit, si l'on compare le héros chamissonien - un homme médiocre qui

7. D. Rabaté, Le Chaudron fêlé - Écarts de la littérature, José Corti, coll. «Les Essais», 2006, p. 70.

8. A. von Chamisso [18I4], L'Étrange Histoire de Peter Schlemihl, trad. M.-Fr. Azéma, Bordas, coll. «Livre de Poche», I995. 
s'enrichit grâce à la bourse inépuisable qu'il a échangée avec le diable contre sa propre ombre - avec Molieri qui devient célèbre en sacrifiant sa voix. Le motif de l'ombre relève, chez Chamisso, du thème du double, cher au romantisme allemand, tout comme la voix peut prétendre être une variante de ce thème. Dans les deux récits, l'improbable se produit : par une intervention surnaturelle s'opère une séparation entre la personne et son double. Cette aliénation apporte la gloire et la richesse. Mais ce gain ne découle en vérité que de la malédiction. Dans le récit de des Forêts, plus la voix devient puissante et occupe le devant de la scène, plus le personnage s'efface pour devenir «insignifiant ${ }^{\natural} »(G M C$, p. 34). La gloire va de pair avec le sacrifice dont l'enjeu est l'anéantissement de la personne. Dans les deux récits, le contrat avec le diable apporte une gloire qui dépasse non seulement l'attente des héros mais aussi leur propre capacité. Pris de panique et de peur, ils tentent en vain de reprendre en mains la situation. Dans "Les grands moments d'un chanteur», l'aliénation et le déséquilibre s'aggravent à tel point que Molieri se trouve face à un choix ultime : doit-il ou peut-il continuer à se réjouir du succès apporté par la voix devenue surpuissante et surhumaine? Ou bien devrait-il plutôt sauver sa personne en sacrifiant sa voix? Comme le héros de Chamisso qui tente de reprendre son ombre, Molieri décide de détrôner sa voix en la ridiculisant. Mais la fin du récit est plutôt amère, car non seulement il perd sa voix, mais il découvre, dans son combat contre son double, quelque chose d'indigne. Lorsque le narrateur demande au héros s'il ne chantera plus, il répond : "C'est que... Comment me mettre dans la peau de quelqu'un que je ne suis plus ou que je serai peut-être de nouveau, comment me prêter à une telle comédie?» (GMC, p. 57) : La carrière extraordinaire de Molieri ne signifie pour lui qu'une «comédie» ou plutôt une tragédie ironique.

Or, la voix, étant ici «séparée», fonctionnant comme un objet a de Lacan, incite le sujet à la désirer, c'est-à-dire à la désirer comme l'objet qui à la fois lui appartient et ne lui appartient pas. L’ambiguïté du rapport entre la voix et la personne se découvre de manière évidente et l'histoire de Molieri l'exprime sous forme de conte fantastique. N'est-ce pas alors que l'histoire de Molieri calque à l'envers le fameux conte sur la voix écrit par E. T. A. Hoffmann? Il s'agit d'un épisode des Contes d'Hoffmann ${ }^{\mathrm{IO}}:$ Krespel, lors d'un voyage en

9. Molieri vit un phénomène dans lequel sa voix absorbe son existence : «On aurait dit qu'il avait ramassé toutes ses forces pour le chant, que toute vigueur en lui avait disparu qui n'était pas au service du chant» (p. 32-33). Quant à l'«insignifiance» du personnage, ce n'est pas la voix qui le rend «insignifiant». Pour être précis, c'est le personnage qui peut devenir «quelqu'un " grâce à la voix qui lui offre une personnalité même si celle-ci est illusoire.

Io. E. T. A. Hoffmann, «Les frères de Saint-Sérapion» («Le conseiller Krespel»), dans l'Intégrale des contes et récits, éd. A. Béguin et M. Laval, Phébus, coll. «Verso", I98I, p. 69-93. 
Italie, a épousé une cantatrice dont il a eu une fille. Cette enfant, Antonie, a une voix splendide, mais elle est atteinte d'un défaut organique de la poitrine, ce qui fait qu'à chaque fois qu'elle chante, elle perd un peu de la vie qu'il lui reste à vivre. D'où l'angoisse du père, Krespel, qui décide d'écarter de sa fille toute source sonore. De plus, Krespel fait appel à une manigance pour faire croire à sa fille qu'elle peut toujours chanter de sa belle voix. À l'insu d'Antonie, il fait jouer du violon chaque fois qu'elle chante, de telle sorte qu'elle croit s'entendre quand chante le violon. Si ce conte parle avant tout d'une heureuse perméabilité entre sujet et objet - perméabilité qui est pour Hoffmann la qualité qui réside dans la musique -, il peut déceler une identité indécise que possède la voix ainsi que son rapport ambigu au sujet. Autrement dit, il est question ici du statut incertain de la voix déchirée entre le rêve de l'unité et la réalité de la division.

"Car chanter, au sens romantique, c'est cela : jouir fantasmatiquement de mon corps unifié", écrivait Roland Barthes ${ }^{\text {II }}$ La voix fait rêver le sujet à l'unité corporelle alors que cette promesse ne peut que s'appuyer sur un leurre. Le conte d'Hoffmann et celui de des Forêts mettent tous les deux en question le sentiment incertain d'identité qu'on tire de sa propre voix. Comme on le sait déjà, Antonie ne peut obtenir le sentiment d'ipséité par le biais d'une voix qui n'est pas la sienne. Dans le conte romantique, cette division n'est pas encore un signe dangereux puisqu'elle est considérée comme une fusion avec l'autre, tandis que chez des Forêts, elle n'est qu'une source de souffrance. Ce thème du rêve et de l'illusion, exploré à l'époque romantique, se retrouve aussi lié au thème d'automate (thème cher à Hoffmann), en s'interrogeant plus que jamais sur la question de l'identité. Le récit de des Forêts se place dans cette lignée mais expose cette interrogation de manière plus grave et définitive.

Sur ce point, citons un autre conte romantique intitulé Don Juan, écrit par le même E. T. A. Hoffmann et qui a inspiré de manière évidente «Les grands moments d'un chanteur ${ }^{12} »$. L'un et l'autre ont la particularité de mettre en avant le personnage de Donna Anna - personnage mozartien, dame déshonorée et fille du Commandeur assassiné par la main du séducteur - à côté du héros Don Juan. Conte fantastique, Don Juan parle du rêve quasi mystique réalisé par la musique, rêve qui promet l'union entre chanteur, personnage et auditeur. Afin de réaliser l'union fusionnelle de ces trois termes touchés par la musique, Hoffmann brouille la frontière entre la scène où se joue l'opéra Don Giovanni et la réalité où vit le narrateur du récit. Cette

II. R. Barthes, L'Obvie et l'Obtus [I982], Essais critiques III, Seuil, coll. «Essais», I992, p. 255.

I2. E. T. A. Hoffmann, Don Juan, dans l'Intégrale des contes et récits, "Fantaisies dans la manière de Callot», éd. A. Béguin et M. Laval, Phébus, coll. «Verso», I979, p. 99-II2. 
perméabilité de la frontière entre les êtres, malgré la distance qui les sépare, donne au récit le ton déterminant : celui de l'étrangeté. Si cette étrangeté est source de bonheur, c'est parce qu' elle annule ici le sentiment de déchirement dans une unité nouvelle qu'est la musique.

Dans le récit de des Forêts se retrouve le même brouillage de la frontière entre la réalité et le rêve, mais ce brouillage, loin d'aboutir à une fusion, ne peut engendrer que des confusions et catastrophes. Molieri, hors scène, continue à se comporter comme un Don Juan réduit à une médiocrité scandaleuse. Et, lorsque ce même Molieri doit jouer le rôle de Don Giovanni sur scène, il traîne cette image désenchantée. La confusion est totale et le récit de des Forêts ne montre la réalité que comme un reflet malveillant du rêve suscité par la musique. Bien plus, incapables de distinguer la réalité du rêve, les autres protagonistes sont contraints à vivre dans un monde de leurre. De même que le narrateur, suspendu dans l'enchantement musical, Anna Fercovitz prend l'illusion pour la réalité en prenant Molieri pour un don Juan. Cette Anna Fercovitz est une caricature de Donna Anna, personnage sublimé dans le conte d'Hoffmann. Dans Don Juan, le personnage de Donna Anna est une médiatrice qui, par sa présence mystérieuse et omniprésente, conduit le narrateur dans la partie la plus intime de la musique mozartienne. Anna Fercovitz, chez des Forêts, s'obstine à assimiler le chanteur au personnage qu'il joue sur scène. Loin de pouvoir toucher au sentiment profond offert par la musique, elle est en proie à une apparence trompeuse. De plus, elle croit aimer la personne de Molieri alors quelle n'aime que sa voix. La confusion éclate à la fin du récit, lorsque Molieri a quitté la scène musicale après le sabotage définitif.

Regardez! Là! Devant nous! Une veuve!», tel aurait été, selon une anecdote dont l'authenticité importe peu, le propos singulier tenu par Molieri un jour qu'attablé à la terrasse d'un café en compagnie d'un ami [...] il vit déboucher sur le trottoir d'en face, très lentement, les yeux perdus dans un sombre rêve, la tête comme alourdie par le poids d'un chagrin sans remède, Anna Fercovitz, Anna éclatante et funèbre comme le plus somptueux chrysanthème, Anna à qui une belle voix avait été si chère qu'elle semblait en porter le deuil. (GMC, p. 62.)

Le lecteur sentira dans ce passage non seulement une dénonciation de l'illusion causée par un effet musical, mais aussi un hommage presque douloureux qu'un auditeur peut exprimer envers une musique adorée. La musique apparaît en ce sens comme un deuil impossible. Mais revenons au cas d'Anna Fercovitz qui dit être plus amoureuse de la voix que de la musique. C'est ce point qui permet de rapprocher de nouveau le récit de des Forêts de celui d'Hoffmann dans lequel le thème de la voix est relié à la promesse d'unification du corps rêvé. Si Anna Fercovitz porte le deuil de la voix de 
Molieri et non de sa personne, c'est parce qu'Anna s'unissait à Molieri par le truchement de la voix de ce dernier. Sur cette dimension symbolique de la voix, Dominique Rabaté donne une explication :

Cette jouissance du corps unifié, je dirai pour ma part que c'est ce que la voix promet, se promet. Si je mets ainsi en avant la dimension de la promesse, c'est pour désigner la part de leurre imaginaire de cette promesse, avec ses effets de conviction et de séduction. La dimension de leurre de la voix explique son extraordinaire pouvoir de fascination, son impact proprement érotique. La thèse que je voudrais maintenant défendre est que la littérature (ou l'opéra) nous dévoile justement cette puissance de leurre, cette structure de promesse de réconciliation et d'unification de la voix, en en faisant miroiter les effets, tout en les dénonçant, tout en les mettant à distance. (Rabaté, p. 68.)

On peut dire qu'avec le récit de des Forêts, le discours sur la musique passe de la description idéalisée des phénomènes musicaux à l'analyse plus distanciée du dispositif musical. Cela revient à reconnaître le rôle symbolique que joue la voix au cœur de l'affaire. Comme le souligne Dominique Rabaté, l'opéra est un lieu de déchirement entre la promesse et la déception, entre l'enchantement et la désillusion. Et l'auditeur reste comme suspendu dans le rêve de promesse dont la voix merveilleuse est un signe avant-coureur. Ce qui est plus paradoxal ici, c'est que plus on est proche de cette promesse, plus on est trompé dans le leurre, l'opéra a ainsi besoin de surenchère psychologique et de surthéâtralité pour maintenir cette promesse. C'est ce dispositif de leurre que le récit de des Forêts met en lumière en racontant l'étrange aventure de Frédéric Molieri.

\section{L'opéra : un leurre ou une gloire manquée?}

Il est temps de remettre la voix dans son contexte qu'est l'opéra. Qu'est-ce que la voix de l'opéra? Roland Barthes, en tentant de distinguer la voix de lied de la voix de l'opéra, qualifie cette dernière de personnalisée, historique, extériorisée et sexuée. Dans l'opéra, les voix ${ }^{13}$ (basse, ténor, soprano, contralto) ont les mêmes titres que des personnages, marqués sexuellement et chargés d'éléments dramatiques. (Il est donc logique qu'Anna Fercovitz prenne la voix de Molieri pour un organe sexuel ${ }^{14}$.) La voix dans l'opéra ne peut pas

13. Dans l'opéra, il s'agit des voix et rarement d'une seule voix, étant donné qu'elles font office des personnages. En ce sens, l'histoire des «Grands moments d'un chanteur» ne peut que susciter le sentiment d'étrangeté puisqu'elle se passe comme s'il s'agissait d'une seule voix, surpuissante, qui éclipse tout le reste et devient l'objet de la fantasmagorie. L'auteur nourrit cette illusion en donnant à la voix de Molieri un caractère mégalomaniaque qui prétend recouvrir l'étendue de toutes les voix et pouvoir jouer ainsi en même temps Othello, Don Giovanni, Gaspar ou même Carmen ou Lulu. Voir GMC, p. II.

I4. Voir $G M C$, p. 60. 
rester neutre, elle n'est pas non plus un simple «instrument», serviteur de la musique comme l'a pensé Balzac ${ }^{15}$. L'opéra apparaît comme un lieu d'imbrication où plusieurs éléments sont en jeu. Comme le rappelle la définition de l'Encyclopédie de Diderot et d'Alembert, l'opéra est «la représentation d'une action merveilleuse $[\ldots]^{16} »$. Né d'une hybridation de musique et de théâtre, l'opéra se pare en vérité d'un caractère obscur :

On devine que la transition est souvent insensible entre l'enchantement au sens littéral [...] et l'enchantement au sens figuré, «l'extase, l'enthousiasme, l'ivresse des sentiments» [...]. Un opéra sans divinité ni sorciers, mais où les passions sont nobles et grandes, peut encore répondre à l'attente d'enchantement (Starobinski, p. I8.)

Dans l'opéra, il n'y a pas de séparation morale entre l'enchantement magique et le leurre, puisque les spectateurs y sont tous "victimes consentantes d'un même mirage" (GMC, p. 20).

L'opéra est un espace de désir dans lequel, avide, le spectateur cherche une proie à laquelle il veut s'identifier. La voix du chanteur transforme, par sa puissance magique, le "travesti» en un héros, en même temps que la "fiction» en un mythe. Le récit de des Forêts montre bien ce qui maintient la fascination musicale : même si l'émotion engendrée par la voix est vraie, elle est largement amplifiée aussi bien par le désir du spectateur que par l'effet de la représentation.

Or, le spectateur n'est pas ici le seul qui soit trompé. Contraint à jouer les rôles acclamés par le public, le chanteur se trouve violemment détourné de sa propre personne. Il est pour ainsi dire piégé dans un monde factice, puisqu'on lui donne une fausse identité. À travers les représentations - de Don Giovanni à Othello -, la tentative d'identification de Molieri échoue au moment même où son existence cherche à s'affirmer dans sa voix. L'aliénation de l'être pour Molieri est donc inhérente au système de représentation. D'où le désir du chanteur de cesser sa carrière pour dénoncer «l'inutilité vaniteuse de la représentation" (Roudaut, p. I55). Molieri, "un homme dépossédé» $(G M C$, p. 45$)$, recourt alors à sa seule arme et l'utilise pour que l'illusion se retourne contre elle-même et qu' elle ouvre la faille :

Frédéric Molieri, après une mystérieuse odyssée intérieure dont nous ne savons rien, punit ceux qui l'avaient aimé pour ce qu'il n'était pas, bafoua le culte qu'il avait suscité, fit voler en éclats sa propre renommée et sombra dans un formidable naufrage au milieu de la colère d'un public venu pour l'acclamer. (Ibid.)

Il tente ainsi de démolir, de l'intérieur, le système d'illusion.

I5. Voir par exemple, H. de Balzac, Massimilla Doni, dans La Comédie humaine, t. X, op. cit., p. 612-613.

I6. Cité par J. Starobinski, Les Enchanteresses, Seuil, coll. «La Librairie du Xxi ${ }^{e}$ siècle», 2005, p. I7-I8. 
Si Molieri ne peut pas s'approprier sa voix, c'est parce qu'il est contraint chaque fois de passer par les "personnages» de fiction, Don Juan, Othello ou encore Gaspar... On découvre ici un décalage presque tragique par rapport au Don Juan d'E. T. A. Hoffmann. Chez des Forêts, la musique est comme engloutie dans le système d'illusion dont le chanteur, comme l'auditeur, est victime.

Afin de souligner cet aspect illusoire, l'écrivain transforme son récit en un monde dans lequel tout se dédouble : la réalité n'est que la déformation du «mirage»; Molieri n'est qu'une caricature médiocre de Don Juan; l'auditeur vit dans le monde dédoublé où la réalité ne s'oppose plus au rêve... L'art de des Forêts consiste à transformer, par le biais de la fiction, le phénomène musical en une sorte de drame psychologique, comme le montre aussi ce commentaire de Jean Roudaut :

[...] la vérité, en littérature, ne dépend pas de l'ordre de la narration, mais du respect des contradictions, par le moyen d'énoncés simultanément valables. La fiction, exploitant consciemment son pouvoir de miroir, ne constitue pas un éloignement de la vérité; elle est la seule forme oblique par quoi puisse s'en formuler le désir.» (Op. cit., p. 133.)

Voyons maintenant de près ce système de leurre. Comment est-il décrit dans le récit et comment notre héros tente-t-il de restituer son statut de personne à part entière? Il est ici intéressant de rappeler le début de Molieri sur la scène lyrique. Hautboïste de formation, sa place est dans l'orchestre jusqu'au jour où un chanteur célèbre déclare forfait pendant la représentation de Don Giovanni, et il incombe à Molieri de remplacer le rôle-titre, car on sait qu'il peut imiter ce chanteur à s'y méprendre. Il monte alors sur scène en tant que "doublure» (GMC, p. I4) d'une voix qui n'est pas la sienne, et, et en tant que "quelque obscur suppléant» $(G M C$, p. 19), il obtient son premier succès. Cet épisode montre avec ironie l'initiation du héros au monde de l'illusion qu'est l'opéra. Une fois dedans, il découvre que le leurre est maintenu par la dépendance réciproque entre le chanteur et son auditeur. Si la voix puissante du héros captive et maintient l'auditeur dans l'enchantement, c'est au prix d'incarner l'image idéalisée que chaque auditeur a de lui-même et qu'il projette sur les personnages par le biais de la voix chantée.

Contraint à incarner l'image que le désir de l'autre projette sur lui, le chanteur en est prisonnier. Comme nous l'avons déjà vu, l'opéra signifiait pour Molieri le lieu où se joue l'identité de sa personne par le truchement de sa propre voix. Il se trouve alors piégé entre le désir de l'autre et le sien, il lui faut à tout prix sortir de ce monde de leurre. Mais comment? Il trouve un moyen simple et efficace qui est de détruire l'image - l'aspect visuel de la représentation - des personnages qu'il joue dans différents rôles. C'est pour 
une raison profonde que Molieri s'attaque surtout à l'image de Don Juan, ce dernier étant une icône sublime et mythique, héros bien au-delà d'un séducteur hors du commun. Si Don Juan se distingue dans le récit de des Forêts, c'est à cause non seulement de son caractère solitaire et sublime, mais à cause surtout du fait qu'il reste maître de lui, contrairement à Molieri, esclave de l'illusion. C'est donc pour une double raison - pour détruire l'image nourrie de leurre et pour vaincre Don Juan apparaissant comme son altérité idéalisée - que Molieri s'attaque à l'image du Maître suprême. Grâce à la manipulation du chanteur, le personnage de Don Juan est abaissé, réduit à l'image de double dégradé de son minable valet Leporello (ici, ce n'est pas le valet qui imite son maître, mais le maître qui imite son valet). Ce détournement apparaît alors comme un retournement complet par rapport à l'interprétation traditionnelle dont témoignent les pages de Kierkegaard sur Don Giovanni de Mozart :

Avec une vraie génialité Mozart fait en somme reproduire Don Juan par Leporello, et obtient ainsi deux résultats : l'effet musical d'entendre Don Juan partout où Leporello est seul présent et, quand tous deux sont en présence, l'effet parodique d'entendre Leporello faire écho à Don Juan et par cela le parodier inconsciemment ${ }^{17}$.

Frédéric Molieri joue Don Juan en parodiant consciemment Leporello, comme si par ce moyen, le chanteur voulait démontrer qu'il est un bouffon et ne pouvait mettre en scène que son anéantissement. Pour parvenir à la destruction de son rôle, il suffit à Molieri de le priver de sa dimension tragique, de la conscience du péché» (Roudaut, p. 153.)

C'est ainsi qu'au premier acte il se bornera à déformer de plus en plus grossièrement l'allure, le caractère de son personnage dont il fera un frère jumeau de Leporello, une sorte de bouffon libidineux, un coquin plat et cruel, une fripouille dépourvue de scrupules et que n’atteint jamais la conscience du péché. ( $G M C$, p. 46.).

Le but de Molieri est de transformer le personnage de Don Juan en «une mauvaise effigie de lui-même» (ibid.). Le scandale éclate et le chanteur réussit son pari de faire

[...] descendre Don Juan degré par degré jusqu'au niveau le plus bas de l'abjection, là où le crime ne se pare d'aucune noblesse, d'aucun courage et ne peut inspirer que le dégoût». (GMC, p. 46-47.)

Il reste à savoir si le chanteur a pu obtenir le sentiment d'unité de soi en immolant son héros. La réponse est négative, car il ne lui suffisait pas de salir l'image de Don Juan pour démolir le personnage. Molieri découvre alors que la musique de Mozart, sublime et intacte, résiste à sa tentative de démolition.

I7. S. Kierkegaard, Ou bien... Ou bien... [1943], traduction de F. et O. Prior et M. H. Guignot, Gallimard, coll. «Tel», 2005, p. IO2. 
Mais châtrer son personnage, le priver de sa grandeur solaire, donner à tous ses actes et jusqu'à celui du défi suprême un caractère hybride à mi-chemin de la lâcheté et de l'arrogance, Molieri n'y pouvait réussir qu'en s'attaquant à la musique même de Mozart. (GMC, p. 47.)

Puisqu'il est impossible dans l'opéra de séparer la représentation de la musique, la démolition de Don Juan signifie avant tout l'anéantissement de l'opéra dans son ensemble. Cela signifie pour Molieri sacrifier sa carrière et nier la puissance de sa voix, autrement dit, consentir à utiliser sa voix merveilleuse au service non pas de la musique mais de sa destruction. Ce que Molieri va tenter n'est rien d'autre que l'extermination de soi c'est-à-dire celle du recouvrement de son identité, en même temps que l'extermination de la musique, c'est-à-dire la chose la plus importante pour le chanteur. Molieri peut alors atteindre au sentiment complet de soi par un moyen unique, en se sacrifiant totalement, de manière suicidaire, dans la musique. La dernière partie du récit est consacrée à la scène de ce suicide étrange, une sorte de destruction réciproque entre le chanteur et la musique. C'est à travers cette scène que se révèle aussi une relation autrement intime entre Don Juan et Frédéric Molieri. Le sabotage suicidaire du chanteur crée, dans le système de leurre, des fissures qui jettent une lumière, quoique faible, sur ce que constitue le mystère central du récit : Qui est Frédéric Molieri? Qu'est-ce que la musique?

Avant d'analyser la fin du récit, je me propose de faire un petit détour autour de la figure de Don Juan, non pas en tant que personnage mythique mais en tant que personnage incarné dans la musique de Mozart. Sans citer la célèbre phrase de Kierkegaard - «La nature de Don Juan est musique» -, beaucoup de penseurs ont noté l'affinité entre le personnage et la musique. Mozart lui-même, avec la complicité de son librettiste Da Ponte, prête à Don Juan l'image de l'excès en le faisant s'exprimer par la musique qui circule librement dans l'espace de l'opéra, cela est tout aussi vrai, même si nos oreilles contemporaines n'y trouvent que la beauté classique. Cet excès qui caractérise le personnage de Don Juan provient de sa capacité de jouissance, de son énergie érotique qui ne lui fait rechercher que fêtes et plaisirs, comme le prouve cette phrase de Georges Bataille, "Don Juan n'est [...] qu'une incarnation personnelle de la fête, de l'heureuse orgie qui nie et divinement renverse les obstacles ${ }^{18}{ }^{\prime}$. Un autre commentateur célèbre de Don Giovanni, Kierkegaard, associe la nature de Don Juan au mal. Mais, si ce personnage est la figure du mal, comment ce mal peut-il être incarné dans un seul personnage alors qu'il doit être protéiforme de nature? Le mal, selon le philosophe,

\section{I8. G. Bataille, L’Expérience intérieure, Gallimard, I943, p. I.}


correspond à l'Éros du monde grec, Éros en tant que vie solaire, sans ombre de culpabilité. En suivant Pierre Klossowski qui donne un éclairage sur l'idée sinueuse de Kierkegaard, on comprend que cet Éros était partout présent chez les Grecs sous des formes multiples sans qu'il éprouve le besoin d'être représenté ni conceptualisér ${ }^{19}$. L'Éros remplissait la vie chez les Grecs. Alors vint le christianisme qui a

[...] introduit dans le monde l'idée d'incarnation ou de représentation : une figure individuelle, en représentant ou en incarnant un principe, en concentre la force à laquelle un chacun participe en contemplant cette figure. Dès lors, la conscience chrétienne a pu également concevoir des figures qui incarnaient les principes et les forces qu'elle exclut. [...] Kierkegaard ne pouvait en son temps connaître la signification des mystères dionysiens. À plus forte raison devait-il être porté de par sa nature à chercher l'élément dionysiaque dans le monde de la sensibilité chrétienne, à le pressentir et à le trouver en l'occurrence dans l'œuvre exaltante de Mozart. (Klossowski, p. 15.)

Ainsi, ce qui est de nature irreprésentable est représenté sous forme de concept. Et puisque l'Éros n'était pas désiré par la pensée chrétienne, il est condamné comme mal, mal nécessaire en quelque sorte, réduit à un concept, à l'opposition au bien et représenté entre autres par la figure de Don Juan. On comprend alors pourquoi Kierkegaard apprécie particulièrement le mal chez Don Juan incarné dans l'opéra de Mozart. Kierkegaard voyait, si j'ose dire, la représentation du mal moins dans la figure de Don Juan elle-même que dans la musique consacrée à son mythe. Ainsi écrit Klossowski :

La génialité sensuelle est tout entière force, tempête, impatience, passion; elle est quelque chose d'essentiellement lyrique : cependant elle ne consiste pas en un moment mais en une succession de moments... d'où son caractère épique : elle est trop débordante pour qu'elle puisse s'exprimer par le mot : elle se meut constamment dans l'immédiat... [...] Cette idée de Don Juan est d'autant plus musicale que la musique ne s'y exprime pas comme accompagnement, mais comme la révélation de son essence la plus intime. (p. I8-I9)

Le mal selon Kierkegaard n’a rien de moral, mais il désigne l'énergie créatrice dont la musique de Mozart est une parfaite illustration. "Don Juan est la forme suprême des métamorphoses de l'immédiat érotique telles que Mozart les a révélées à Kierkegaard» (p. 20).

C'est à ce mal dont Don Juan est la figure sublime - mal en tant que figure de devenir et de force destructrice pure (c'est pourquoi Don Juan ne peut pas s'arrêter à une seule passion, il est condamné à passer des unes aux autres) - que Molieri répond d'une manière étrange dans la dernière scène du récit. Au cours du sabotage qui massacre impitoyablement la musique de

19. P. Klossowski, «Don Juan selon Kierkegaard», dans Tableaux vivants, Essais critiques 1936-1983, Gallimard, coll. «Le Promeneur», 200I, p. I3-24. 
Mozart ( $«$ il n’hésitera pas, pour finir, à user de la seule arme dont il dispose encore; il chantera faux, faux à faire crier les sourds» [GMC, p. 47]), s'opère une étrange communion du mal entre la voix de Molieri et la musique de Don Giovanni à moitié massacrée. Même au milieu d'une anarchie débordante, le chant de Molieri est en quelque sorte préservé de la destruction, bien capable encore d'exercer une fascination sur son auditeur. Malgré le désordre ou grâce à lui, la voix de Molieri manifeste un éclat tout particulier. Citons le témoignage du narrateur :

Je n'étais pas du nombre des spectateurs avertis qui éprouvèrent dès les premières scènes une sorte de gêne dont ils rendirent d'abord responsable leur mauvaise disposition personnelle ou l'acoustique défectueuse de la salle : c'est peut-être que Molieri recommençait à exercer sur moi une fascination qui me rendait inattentif aux insuffisances de sa voix, aux entorses de plus en plus flagrantes qu'il faisait subir à la partition en s'excitant à fournir des records injustifiables. [...] Mais au milieu de ce débordement d'anarchie, et réduit à l'état de pantin, Molieri continuait à m'en imposer par ce qu'il y avait en lui d'affreusement énergique et de troublant. [...] Il réussissait à donner au spectacle de sa déchéance qui revêtait ici les apparences d'un sacrifice librement consenti et comme d'une immense liquidation - le même caractère de cérémonie magique et soigneusement préparé que deux ans plus tôt à celui de sa fulgurante accession à la gloire, et sa voix ellemême, bien qu'elle eût perdu sa pureté, sa justesse, sa puissance, ne m'en paraissait que plus émouvante, telle la voix d'une vieille chanteuse, autrefois célèbre, d'où remonteraient comme d'un très lointain passé les accents qui nous avaient bouleversés au temps où l'âge ne l'avait pas encore fêlée. (GMC, p. 45-49.)

Il est vrai que ce geste de massacre musical a la valeur pour le chanteur de sacrifice de soi. Cependant, Molieri n'est pas ici un simple perdant, puisque dans cet acte d' "immense liquidation", il réussit à se libérer des images fausses que l'autre projetait sur lui. Le spectateur assiste alors à une sorte de mise à nu où surgit une étrange puissance qui circule sur scène. Le «mal» jusque-là dissimulé derrière l'apparence de la représentation revient alors à la surface :

"Ah, dov'é il perfido, dov'e l'indegno? Tutto il mio sdegno sfogar io vo!», entonne le quatuor des accusateurs à la scène finale qui constitue comme la moralité du drame: on rit, on applaudit, de même qu'on souligna par des murmures approbateurs les paroles d'Elvire reprises ensuite par le chour: "Ah! certo è l'ombra che l'incontro!» Mais Molieri n'avait-il été vraiment en la circonstance que l'ombre de lui-même? Ne pouvait-on dire au contraire qu'il n'avait jamais révélé aussi publiquement et en le poussant jusqu’au paroxysme, ce qui faisait le fond de sa nature puissante : le goût de la subversion et l'horreur du mensonge dont il se reprochait d'avoir été jusque-là le serviteur trop respectueux? Car cette insurrection systématique, il l'avait d'abord dirigée contre lui-même, quel que fût le prix dont il faudrait la payer et le risque à encourir de ce fait; les surprenantes aliénations de sa voix, ses discordances affreuses n'étaient-elles pas comme les symptômes d'un mal plus intime qu'il avait décidé d'exposer aux feux de la rampe, non pas seulement dans une intention blasphématoire, mais 
afin de bousculer l'odieux édifice de sa renommée, afin de se perdre sans recours dans l'opinion de ceux qui le louaient pour ce qui ne méritait pas l'éloge et ne l'aimaient que pour ce qu'il n'était pas? (GMC, p. 50.)

Il n'y a pas de doute que le "mal» se mette au devant de la scène au fur et à mesure que la musique s'efface. Mais, en vérité, on n'en sait pas davantage sur ce mal, à savoir d'où il vient ou de quelle nature il est ${ }^{20}$. On peut certes souscrire à l'hypothèse du narrateur ici émise pour attribuer ce mal à la vraie nature de Molieri qui éclate au moment même où le travail de démolition bat son plein. En admettant que ce "mal» appartienne au chanteur - ce qui n'est pas certain ${ }^{21}-$, il reste à savoir si cette manifestation du mal peut être comprise comme une affirmation complète de la personne de Molieri enfin réalisée, ou bien au contraire si elle est un signe de la négation de soi que le chanteur achève en surjouant le rôle qui lui est attribué, c'est-à-dire un Don Juan cette fois totalement livré à lui-même (puisque Molieri rêvait de jouer un personnage masculin qui soit "à la fois l'instrument du mal et sa victime $\left.{ }^{22} »\right)$ ? Dans ce cas, le mal vient-il plutôt de la musique qui se révèle dans sa forme brute, privée de toute beauté superficielle, dans laquelle Molieri se noie et se suicide en tant que chanteur? Le récit de des Forêts ne donne évidemment aucune réponse à toutes ces hypothèses (C'est en cela un conte sans moralité). Il préfère laisser ainsi un sentiment de vide, gravé comme une blessure mortelle dans le cœur de l'auditeur ${ }^{23}$.

Dans «Sarrasine», Balzac fait dire à son héros à l'adresse de sa chanteuse adorée : «Tu n’es rien» (p. I074). Ces mots révélateurs ne sont pourtant pas prononcés dans une scène de l'opéra, comme si l'écrivain voulait lui-même éviter d'affirmer ce vide musical derrière l'apparence de représentation, afin de maintenir malgré tout sa foi artistique selon laquelle l'art doit représenter. La littérature reste ainsi discrète sur ce "rien" jusqu'à ce que Mallarmé en fasse l'éloge. Le «rien" devient alors un objet d'adoration. C'est à cette idée sublimée du «rien» musical que le récit de des Forêts oppose le sentiment de haine et de malaise. La musique fait jouer à Molieri tout ce qu'il possédait. Il en sort plus démuni que jamais, laissant derrière lui le souvenir d'une voix superbe et inoubliable.

20. L'ambivalence du «mal» est bien le point névralgique de ce récit et il nous faudra y consacrer une autre étude.

2I. Voir $G M C$, p. II-I2.

22. Voir $G M C$, p. 34.

23. Cette blessure est directement reliée à la motivation du narrateur de connaître la vérité sur Molieri et on peut considérer qu'elle est le point de départ même du récit. 
